Research Article

\title{
Classical and Quantum Models of Diffusion
}

Angelo Morro *

DIBRIS, University of Genoa, Via All'Opera Pia 13, 16145 Genoa, Italy; E-Mail:

angelo.morro@unige.it

* Correspondence: Angelo Morro; E-Mail: angelo.morro@unige.it

Academic Editor: Vardan Apinyan

Special Issue: Quantum Mechanics in Solid State Systems

Recent Progress in Materials

2021, volume 3, issue 2

doi:10.21926/rpm.2102011
Received: February 19, 2021

Accepted: March 26, 2021

Published: April 13, 2021

\begin{abstract}
The objective of the paper is twofold: first, to review the classical diffusion models and show the approximations at the origin of the parabolic character of the classical equations; second, to demonstrate a connection between the quantum and classical models of diffusion. As diffusion is inherently related to the motion of constituents, the consistent models are framed within the dynamics of mixtures. The derivation of diffusion equations is then determined based on the related, pertinent approximations.
\end{abstract}

\section{Keywords}

Diffusion equations; diffusion flux; chemical potential; mass fractions

\section{Introduction}

Diffusion phenomena are modeled by several differential equations, and classical approaches to diffusion are considered both interesting and a useful reference in quantum models. In classical physics, diffusion is naturally framed within the realm of continuum physics, and the theory of conditions of the Creative Commons by Attribution License, which permits unrestricted use, distribution, and reproduction in any medium or format, provided the original work is correctly cited. 
mixtures is considered an essential framework. In this paper, we review some well-known models of diffusion and report the schemes and approximations at the origin of the derivation in some approaches as discussed in the literature.

Though various approaches are discussed in the literature, the best-known equation of diffusion is

$$
\dot{\omega}=D \Delta \omega+\xi
$$

where $\omega$ is the mass fraction (or concentration), $D$ is the diffusivity, and $\xi$ is a given function of $\omega$. Equation (1) is parabolic in character and follows the well-known Fick's law for the mass flux. Parabolic equations in space-time are associated with an infinite speed of the propagation of the initial datum, similar to the standard heat equation. Even in a non-relativistic context, from a physical viewpoint, this feature is often considered an apparent paradox.

Diffusion is quite often observed to be governed by parabolic equations in both classical and quantum models, except for, of course, the steady-state regimes [1]. The parabolic character follows from seemingly different starting schemes as shown, e.g., by the Cahn-Hilliard and AllenCahn equations [2]. Furthermore, phase-field models for alloy solidification [3], coupling between mechanical loading and chemical reactions [4], film growth by vapor deposition [5], and diffusion based on the Maxwell-Stefan approach [6] lead to parabolic equations.

In essence, this paper has two main objectives: at first, to review the classical diffusion models and show the approximations at the origin of the parabolic character of the classical diffusion equations and then establish a relation between the quantum and classical models of diffusion. Generally, it has been observed that diffusion is inherently related to the motion of constituents. Consequently, from the physical viewpoint, consistent models should be framed within the dynamics of mixtures. In this paper, it is described in detail and the derivation of diffusion equations is determined based on the pertinent approximations.

\section{Quantum Derivation of Balance Equations}

If a quantum particle moves in free space, the wavefunction $\psi$ evolves in time according to the Schrödinger equation

$$
i \hbar \partial_{t} \psi=\left(-\frac{\hbar^{2}}{2 m} \Delta+U\right) \psi
$$

where $m$ is the mass of the particle, $\Delta=\nabla^{2}$ is the Laplacian operator and $U$ is the potential of an applied force field. As the wavefunction $\psi$ is complex valued, it can be represented in the polar form as shown below:

$$
\psi=\sqrt{\rho} \exp (i S / \hbar)
$$

where $\rho$ and $S$ are the functions of the position $x$ and time $t$. Of course,

$$
\psi \psi^{*}=\rho
$$


ascribes to $\rho(\mathbf{x}, t)$, that is, the probability density, per unit volume, of finding the quantum particle at the point $\mathbf{x}$ at time $t$. The ratio $S / \hbar$ denotes the argument of $\psi$. Upon evaluation of $\partial_{t} \psi$ and $\Delta \psi$, it follows from Equation (2) that

$$
\begin{gathered}
i \hbar\left[\frac{1}{2} \rho^{-\frac{1}{2}} \partial_{t} \rho+\rho^{\frac{1}{2}} \frac{i}{\hbar} \partial_{t} S\right]=U \rho^{\frac{1}{2}} \\
-\frac{\hbar^{2}}{2 m}\left[-\frac{1}{4} \rho^{-\frac{3}{2}}(\nabla \rho)^{2}+\frac{1}{2} \rho^{-\frac{1}{2}} \Delta \rho+\frac{i}{\hbar} \rho^{-\frac{1}{2}} \nabla \rho \cdot \nabla S-\frac{\rho^{\frac{1}{2}}}{\hbar^{2}}(\nabla S)^{2}+\frac{i}{\hbar} \rho^{\frac{1}{2}} \Delta S\right] .
\end{gathered}
$$

Observe that $\rho^{-1 / 2} \nabla \rho \cdot \nabla S+\rho^{1 / 2} \Delta S=\rho^{-1 / 2} \nabla \cdot(\rho \nabla S)$. By equating the imaginary parts of Equation (3), we obtain the following expressions:

$$
\partial_{t} \rho=-\nabla \cdot(\rho \mathbf{v}), \quad \mathbf{v}:=\frac{1}{m} \nabla S
$$

Equation (4) coincides with the classical continuity equation of continuum physics, where $\rho$ is the mass density and $v$ is the velocity. The real parts of Equation (3) result in

$$
\partial_{t} S=-\frac{1}{2 m}(\nabla S)^{2}+\frac{\hbar^{2}}{2 m}\left[\frac{1}{2} \rho^{-1} \Delta \rho-\frac{1}{4} \rho^{-2}(\nabla \rho)^{2}\right]-U
$$

In view of the identities

$$
\begin{gathered}
\nabla(\nabla S)^{2}=(\nabla S \cdot \nabla) \nabla S \\
\frac{1}{2} \rho^{-1} \Delta \rho-\frac{1}{4} \rho^{-2}(\nabla \rho)^{2}=\rho^{-1 / 2} \Delta \rho^{1 / 2}
\end{gathered}
$$

we can apply the gradient operator to Equation (5), replace $\nabla S / m$ with $\mathbf{v}$, and obtain

where

$$
\partial_{t} \mathbf{v}+(\mathbf{v} \cdot \nabla) \mathbf{v}=-\frac{1}{m}[\nabla Q+\nabla U]
$$

$$
Q=-\frac{\hbar^{2}}{m \rho^{1 / 2}} \Delta \rho^{1 / 2} .
$$

Equation (6) can be considered as the equation of motion per unit mass ${ }^{1}$. In relation to the equation of motion of fluids,

$$
\partial_{t} \mathbf{v}+(\mathbf{v} \cdot \nabla) \mathbf{v}=-\frac{1}{\rho} \nabla p+\mathbf{b},
$$

the left-hand side shows the classical form of the Lagrangian or the total time derivative of $\mathbf{v}$, that is, the derivative relative to the observer moving with the point under consideration, and $\partial_{t} \mathbf{v}+$ $(\mathbf{v} \cdot \boldsymbol{\nabla}) \mathbf{v}$ is the acceleration; the right-hand side shows a body force $\mathbf{b}$ (per unit mass) as $-\nabla U / \mathrm{m}$. The remaining term is similar to a pressure term. Indeed, the function $Q$ is often referred to as the Bohm quantum potential [8].

Concerning the function $U$, and the force $-\nabla U / m$, e.g., as reported in the literature on superconductors $[9,10]$, the effective potential $\tilde{\phi}$ that describes the collective behavior should be $\phi-\mu / e$, where $\phi$ is the electrostatic potential and $\mu$ is the chemical potential. Hence, the

\footnotetext{
${ }^{1}$ In a similar form, Equations (4) and (6) trace back to Madelung [7].
} 
equation of motion (6) comprises a force term $-\nabla \mu / e m$, where $e$ is the charge of electrons. It is interesting to note that a contribution $\nabla \mu$ to the diffusion also occurs in customary classical models.

Diffusion is also modeled on the basis of the Brownian motion that is considered as the microscopic origin. Let $P$ be the probability (spatial) density of the Brownian particles with mass $m$. Hence, from the equation of motion, we have

$$
m \partial_{t}^{2} P+b \partial_{t} P=\nabla \cdot(\nabla \Pi)
$$

where $b$ is the friction coefficient and $\boldsymbol{\Pi}=p \mathbf{1}$ is the pressure tensor, with $\mathbf{1}$ being the identity. In the high friction limit, the inertial term $m \partial_{t}^{2} P$ is neglected as compared to the frictional term. Hence, taking the ideal-gas expression for $\Pi$, it follows that [11]

$$
\partial_{t} P=D \Delta P
$$

where $D=k_{B} \theta / b$ is the Einstein diffusion constant, $\theta$ is the absoulte temperature, and $k_{B}$ is the Boltzmann constant. The quantum effects are then included by adding $[12,13]$

$$
\Pi_{Q}=-\frac{\hbar^{2}}{4 m} P \nabla \nabla P
$$

to the pressure tensor. Then, it follows that

$$
\partial_{t} P=\nabla \cdot\left(\frac{P \nabla Q}{b}+D \nabla P\right)
$$

where $Q$ is the Bohm quantum potential.

Diffusion also means the spreading of a given wave packet. If the probability density is Gaussian,

$$
\rho=\left(2 \pi \sigma^{2}\right)^{-3 / 2} \exp \left(-x^{2} / 2 \sigma^{2}\right)
$$

then Equation (6) is approximated to

$$
m \partial_{t}^{2} \sigma+b \partial_{t} \sigma=\frac{\hbar^{2}}{4 m \sigma^{3}}
$$

Equation (7) describes the evolution of the root-mean-square displacement $\sigma$, and the positive constant $b$ is inserted to induce the particle friction [14]. The increasing function $\sigma(t)$ quantitatively describes the diffusive process via the wavefunction.

With regard to many-particle systems, it is often assumed that the system is governed by a single-particle operator $\rho$ satisfying a modified Liouville equation:

$$
i \hbar \partial_{t} \rho=[\mathcal{H}, \rho]+i \hbar \mathcal{Q}(\rho)
$$

where $\mathcal{H}=-\left(\hbar^{2} / 2 m^{2}\right) \Delta+U$ is the Hamiltonian, [,] denotes the commutator, and $Q$ is a collision operator that describes the interactions between particles [15]. 
The parabolic character of the diffusion equation in quantum contexts appears to be greatly influenced by the classical diffusion equation. In this case, classically diffusion means the relative motion of a constituent in a mixture relative to the other constituents. In order to understand this fact, a brief review of the equations of motion of mixtures is required. For simplicity, we consider fluid constituents, which can be used for charged particles.

\section{Balance Equations for Fluid Mixtures}

Consider a mixture of $n$ fluid constituents [16]. Let the suffix $\alpha=1,2, \ldots, n$ label the quantities related to the $\alpha$ th constituent. The continuity equation of the $\alpha$ th constituent comprises the mass supply $\tau_{\alpha}$, per unit volume, and unit time, so that

$$
\partial_{t} \rho_{\alpha}=\nabla \cdot\left(\rho_{\alpha} \mathbf{v}_{\alpha}\right)=\tau_{\alpha}
$$

The conservation of mass of the mixture implies

$$
\sum_{\alpha=1}^{n} \tau_{\alpha}=0
$$

The mass density $\rho$ and the velocity $v$ of the mixture are defined by

$$
\rho=\sum_{\alpha=1}^{n} \rho_{\alpha}, \quad \mathbf{v}=\frac{1}{\rho} \sum_{\alpha=1}^{n} \rho_{\alpha} \mathbf{v}_{\alpha}
$$

Hence,

$$
\omega_{\alpha}=\frac{\rho_{\alpha}}{\rho}
$$

is the mass fraction (or concentration) of the $\alpha$ th constituent and

$$
\mathbf{u}_{\alpha}=\mathbf{v}_{\alpha}-\mathbf{v}
$$

is the diffusion velocity. Moreover, we have

$$
\sum_{\alpha=1}^{n} \rho_{\alpha} \mathbf{v}_{\alpha}=\rho \mathbf{v}
$$

The sum of Equation (9) over $\alpha$ and use of Equation (10) yield

$$
\partial_{t} \rho=\nabla \cdot(\rho \mathbf{v})=0
$$

which is the continuity equation for the whole mixture, similar to Equation (4).

Replace $\rho_{\alpha}$ with $\rho \omega_{\alpha}$ and $\mathbf{v}_{\alpha}$ with $\mathbf{v}+\mathbf{u}_{\alpha}$ in order to obtain

$$
\omega_{\alpha}\left[\partial_{t} \rho+\nabla \cdot(\rho \mathbf{v})\right]+\rho\left(\partial_{t} \omega_{\alpha}+\mathbf{v} \cdot \nabla \omega_{\alpha}\right)+\nabla \cdot\left(\rho_{\alpha} \mathbf{u}_{\alpha}\right)=\tau_{\alpha},
$$


hence, in view of Equation (11),

$$
\rho\left(\partial_{t} \omega_{\alpha}+\mathbf{v} \cdot \nabla \omega_{\alpha}\right)+\nabla \cdot\left(\rho_{\alpha} \mathbf{u}_{\alpha}\right)=\tau_{\alpha}
$$

In this case, $\partial_{t} \omega_{\alpha}+\mathbf{v} \cdot \nabla \omega_{\alpha}$ is the derivative with respect to the barycentric observer, which is denoted by $\dot{\omega}_{\alpha}$. The vector

$$
\mathbf{h}_{\alpha}:=\rho_{\alpha} \mathbf{u}_{\alpha}
$$

is the $\alpha$ th diffusion flux representing the flux of the $\alpha$ th constituent relative to the barycentric observer [17]. Hence, Equation (12) can be written in the form

$$
\rho \dot{\omega}_{\alpha}+\nabla \cdot \mathbf{h}_{\alpha}=\tau_{\alpha}
$$

The mass fraction $\omega_{\alpha}$ in the barycentric reference evolves according to Equation (14). The evolution equation (14) is made operative by specifying $\tau_{\alpha}$ and $h_{\alpha}$ in terms of $\omega_{\alpha}$ and possibly parameterized by, e.g., the temperature. The equation of motion of the $\alpha$ th constituent is written in the form

$$
\partial_{t}\left(\rho_{\alpha} \mathbf{v}_{\alpha}\right)+\nabla \cdot\left(\rho_{\alpha} \mathbf{v}_{\alpha} \otimes \mathbf{v}_{\alpha}\right)-\nabla \cdot \mathbf{T}_{\alpha}-\rho_{\alpha} \mathbf{b}_{\alpha}=\mathbf{m}_{\alpha}
$$

where $\mathbf{T}_{\alpha}$ is the Cauchy stress tensor, $\mathbf{b}_{\alpha}$ is the body force, and $\mathbf{m}_{\alpha}$ is the growth of the linear momentum, that is, the force on the $\alpha$-th constituent due to other constituents of the mixture. The growth $\left\{\mathbf{m}_{\alpha}\right\}$ is subject to the constraint

$$
\sum_{\alpha=1}^{n} \mathbf{m}_{\alpha}=\mathbf{0}
$$

\section{Classical Diffusion Equations}

The simplest and best known model of diffusion traces back to Fick [18] and is based on an assumption on $\mathbf{h}_{\alpha}$ that follows the analogy of the Fourier model of heat conduction. First, the total derivative $\dot{\omega}_{\alpha}$ is replaced with the partial derivative $\partial_{t} \omega_{\alpha}$; it means that diffusion is described relative to the barycentric observer. Further, Fick's law assumes that the diffusion flux $h_{\alpha}$ is antiparallel to $\nabla \omega_{\alpha}$, i.e., $\mathbf{h}_{\alpha}=-\kappa_{\alpha} \nabla \omega_{\alpha}, \kappa_{\alpha}>0$. Also, divide Equation (14) by $\rho$ and assume $\nabla \rho=$ 0. Hence, we have

$$
\partial_{t} \omega_{\alpha}=\nabla \cdot\left(D_{\alpha} \nabla \omega_{\alpha}\right)+\zeta_{\alpha}
$$

where $\zeta_{\alpha}=\tau_{\alpha} / \rho$ and $D_{\alpha}=\kappa_{\alpha} / \rho$; the quantity $D_{\alpha}$ is called diffusivity. If, instead, $\kappa_{\alpha}$ is assumed to be a constant, $\nabla \kappa_{\alpha}=\mathbf{0}$, then it follows the well-known parabolic equation

$$
\partial_{t} \omega_{\alpha}=D_{\alpha} \Delta \omega_{\alpha}+\zeta_{\alpha}
$$

Other diffusion equations follow the following two main assumptions. Let $\mu_{\alpha}$ denote the chemical potential and $\psi_{\alpha}$ the free energy. The assumptions are

$$
\mathbf{h}_{\alpha}=-\kappa_{\alpha} \nabla \mu_{\alpha}, \quad \mu_{\alpha}=\partial_{\omega_{\alpha}} \psi_{\alpha}-\nabla \cdot \partial_{\nabla \omega_{\alpha}} \psi_{\alpha}
$$


The free energy is taken in the form

$$
\psi_{\alpha}=\left(\omega_{\alpha}, \Delta \omega_{\alpha}\right)=f_{\alpha}\left(\omega_{\alpha}\right)+\frac{1}{2} \lambda_{\alpha}\left|\nabla \omega_{\alpha}\right|^{2} .
$$

For simplicity, let $\lambda_{\alpha}$ be a constant. It follows

$$
\mu_{\alpha}=f_{\alpha}^{\prime}-\lambda_{\alpha} \Delta \omega_{\alpha}
$$

and hence Equation (14) becomes

$$
\dot{\omega}_{\alpha}=\frac{1}{\rho} \nabla \cdot\left(\kappa_{\alpha} f_{\alpha}^{\prime \prime} \nabla \omega_{\alpha}\right)-\frac{\lambda_{\alpha}}{\rho} \Delta^{2} \omega_{\alpha}+\zeta_{\alpha} .
$$

Equation (19) is usually referred to as the Cahn-Hilliard equation $[19,20]$. It is a fourth-order partial differential equation. If the dependence on $\nabla \omega_{\alpha}$ is ignored, then the Cahn-Hilliard equation formally reduces to the second-order parabolic equation (16).

Otherwise, it is assumed that the evolution of $\omega_{\alpha}$ is in fact a relaxation toward equilibrium governed by a parameter $\beta_{\alpha}>0$,

$$
\beta_{\alpha} \dot{\omega}_{\alpha}=-\mu_{\alpha}
$$

Hence, considering that $\psi_{\alpha}$ is given again by Equation (18), we obtain

$$
\beta_{\alpha} \dot{\omega}_{\alpha}=-f_{\alpha}^{\prime}+\lambda_{\alpha} \Delta \omega_{\alpha}
$$

Equation (20) is a second-order partial differential equation when the dependence of $\psi_{\alpha}$ on $\nabla \omega_{\alpha}$ is allowed. The lower order relative to the Cahn-Hilliard equation (19) is interpreted by the fact that Equation (20) describes the ordering of atoms within unit cells on a lattice [21], whereas Equation (19) describes the transport of atoms between unit cells. Equation (20) is referred to as the Ginzburg-Landau equation (see [22]) or the Allen-Cahn [21] equation.

Equations (16), (19), and (20) follow the assumptions $\mathbf{h}_{\alpha}=-\kappa_{\alpha} \nabla \omega_{\alpha}, \mathbf{h}_{\alpha}=-\nabla \mu_{\alpha}$, or $\beta_{\alpha} \dot{\omega}_{\alpha}=$ $-\mu_{\alpha}$. All of them are of parabolic character and, as such, they describe propagation with an infinite speed under transient conditions. We now consider the pertinent differential equation for the mass fractions $\omega_{\alpha}$ in a scheme consistent with the balance equations.

\section{Dynamic Diffusion Equation}

The diffusion process in a mixture is given by the evolution of the mass densities $\left\{\rho_{\alpha}\right\}$. In order to determine these fields, we consider the balance equations of mass and linear momentum, namely,

$$
\begin{gathered}
\partial_{t} \rho_{\alpha}=-\nabla \cdot\left(\rho_{\alpha} \mathbf{v}_{\alpha}\right)+\tau_{\alpha} \\
\partial_{t}\left(\rho_{\alpha} \mathbf{v}_{\alpha}\right)+\nabla \cdot\left(\rho_{\alpha} \mathbf{v}_{\alpha} \otimes \mathbf{v}_{\alpha}\right)=\nabla \cdot \mathbf{T}_{\boldsymbol{\alpha}}+\rho_{\alpha} \mathbf{b}_{\alpha}+\mathbf{m}_{\alpha}
\end{gathered}
$$

Equation (21) represents a system in the $2 n$ unknowns $\left\{\rho_{\alpha}\right\},\left\{\mathbf{v}_{\alpha}\right\}$. The system can be solved once the mass supplies $\left\{\tau_{\alpha}\right\}$, the stress tensors $\left\{\mathbf{T}_{\boldsymbol{\alpha}}\right\}$, and the linear momentum growth $\left\{\mathbf{m}_{\alpha}\right\}$ are given in terms of $\left\{\rho_{\alpha}\right\}$ and $\left\{\mathbf{v}_{\alpha}\right\}$. The body forces $\left\{\mathbf{b}_{\alpha}\right\}$ are assumed to be known. In this scheme, the system (21) is the exact set of equations that describe the evolution of the mixture and hence the 
diffusion. If, though, the constituents are allowed to be viscous and/or heat conducting, then also the balance of energy is considered to account for the temperature.

A simpler form of the system can be obtained by considering some approximations. The time differentiation of the first equation, divergence of the second one, and substitution of $\nabla$. $\partial_{t}\left(\rho_{\alpha} \mathbf{v}_{\alpha}\right)$ yield

$$
\partial_{t}^{2} \rho_{\alpha}=\nabla \cdot\left[\nabla \cdot\left(\rho_{\alpha} \mathbf{v}_{\alpha} \otimes \mathbf{v}_{\alpha}\right)\right]-\nabla \cdot\left[\nabla \cdot \mathbf{T}_{\boldsymbol{\alpha}}\right]-\nabla \cdot\left(\rho_{\alpha} \mathbf{b}_{\alpha}\right)-\nabla \cdot \mathbf{m}_{\alpha}+\partial_{t} \tau_{\alpha} .
$$

Since the constituents are considered as inviscid fluids, we obtain $T_{\alpha}=-p_{\alpha} \mathbf{1}$. It follows

$$
\partial_{t}^{2} \rho_{\alpha}=\nabla \cdot\left[\nabla \cdot\left(\rho_{\alpha} \mathbf{v}_{\alpha} \otimes \mathbf{v}_{\alpha}\right)\right]+\Delta p_{\alpha}-\nabla \cdot\left(\rho_{\alpha} \mathbf{b}_{\alpha}\right)-\nabla \cdot \mathbf{m}_{\alpha}+\partial_{t} \tau_{\alpha}
$$

Let $p_{\alpha}=p_{\alpha}\left(\rho_{\alpha}\right)$, with the temperature $\theta$ being assumed as a parameter. Hence,

$$
\nabla p_{\alpha}=\partial_{\rho_{\alpha}} p_{\alpha} \nabla \rho_{\alpha}
$$

With $\mathbf{m}_{\alpha}, \tau_{\alpha}$ being dependent on the whole set of densities $\left\{\rho_{\beta}\right\}$ and velocities $\left\{\mathbf{v}_{\beta}\right\}$, we obtain a system of equations for $\left\{\rho_{\alpha}\right\}$ and $\left\{\mathbf{v}_{\beta}\right\}$. A simpler system of equations originates by linearizing the dynamic equations (21). Neglect $\nabla \cdot\left[\nabla \cdot\left(\rho_{\alpha} \mathbf{v}_{\alpha} \otimes \mathbf{v}_{\alpha}\right)\right]$ and let

$$
\mathbf{b}_{\alpha}=\mathbf{g}, \quad \mathbf{m}_{\alpha}=\sum_{\beta=1}^{n} M_{\beta \alpha}\left(\mathbf{v}_{\beta}-\mathbf{v}_{\alpha}\right),
$$

where $\mathbf{g}$ is the acceleration gravity and $M_{\alpha \beta}=M_{\beta \alpha}$. We find the system

$$
\begin{gathered}
\partial_{t}^{2} \rho_{\alpha}=\partial_{\rho_{\alpha}} p_{\alpha} \Delta \rho_{\alpha}+\mathbf{g} \cdot \nabla \rho_{\alpha}+\sum_{\beta=1}^{n} M_{\beta \alpha} \nabla \cdot\left(\mathbf{v}_{\beta}-\mathbf{v}_{\alpha}\right), \\
\rho_{\alpha} \partial_{t} \mathbf{v}_{\alpha}=\partial_{\rho_{\alpha}} p_{\alpha} \nabla \rho_{\alpha}-\rho_{\alpha} \mathbf{g}+\sum_{\beta=1}^{n} M_{\beta \alpha}\left(\mathbf{v}_{\beta}-\mathbf{v}_{\alpha}\right)
\end{gathered}
$$

with the unknowns $\left\{\rho_{\alpha}\right\},\left\{\mathbf{v}_{\alpha}\right\}$. If the cross-coupling terms $\mathbf{m}_{\alpha}$ are neglected, then the system decouples, and each equation (for $\rho_{\alpha}$ ) is hyperbolic with the speed of propagation $\sqrt{\partial_{\rho_{\alpha}} p_{\alpha}}$. These equations are the classical sound waves, and we then observe the analogy between the differential equations for the mass densities and the differential equations (26) for the mass fractions.

\subsection{Dynamic Equation for the Mass Fractions}

Equation (14) holds exactly, without any approximation, as a consequence of the balance of mass for the $\alpha$-th constituent of the mixture. The mass flux $\mathbf{h}_{\alpha}=\rho_{\alpha} \mathbf{u}_{\alpha}$ is described by Fick's law as a model equation that is (tacitly) assumed to hold under stationary conditions. Instead, as shown in earlier studies $[23,24]$, the mass flux $h_{\alpha}$ satisfies the rate equation

$$
\dot{\mathbf{h}}_{\alpha}+(\mathbf{L}+\nabla \cdot \mathbf{v} \mathbf{1}) \mathbf{h}_{\alpha}=\nabla \cdot\left(\mathbf{T}_{\boldsymbol{\alpha}}-\rho_{\alpha} \mathbf{v}_{\alpha} \otimes \mathbf{v}_{\alpha}\right)-\frac{\rho_{\alpha}}{\rho} \nabla \cdot \mathbf{T}+\rho_{\alpha}\left(\mathbf{b}_{\alpha}-\mathbf{b}\right)+\mathbf{m}_{\alpha}-\tau_{\alpha} \mathbf{v}
$$


The dynamics of a mixture, at a fixed temperature, is described by the functions $\rho_{\alpha}, \mathbf{v}_{\alpha}, \mathbf{h}_{\alpha}, \omega_{\alpha}$ and is governed by the system of equations (24) along with

$$
\begin{gathered}
\partial_{t} \rho_{\alpha}+\nabla \cdot\left(\rho_{\alpha} \mathbf{v}_{\alpha}\right)=\tau_{\alpha} \\
\partial_{t}\left(\rho_{\alpha} \mathbf{v}_{\alpha}\right)+\nabla \cdot\left(\rho_{\alpha} \mathbf{v}_{\alpha} \otimes \mathbf{v}_{\alpha}\right)=\nabla \cdot \mathbf{T}_{\boldsymbol{\alpha}}+\rho_{\alpha} \mathbf{b}_{\alpha}+\mathbf{m}_{\alpha} .
\end{gathered}
$$

Equation

$$
\rho \dot{\omega}_{\alpha}=-\nabla \cdot \mathbf{h}_{\alpha}+\tau_{\alpha}
$$

follows the continuity equations and the definition of $\rho, \omega_{\alpha}, \mathbf{v}, \mathbf{h}_{\alpha}$. The functions $\mathbf{T}_{\boldsymbol{\alpha}}, \mathbf{m}_{\alpha}$, and $\tau_{\alpha}$ are assumed to be given by the constitutive functions of $\rho_{\alpha}, \mathbf{v}_{\alpha}, \mathbf{h}_{\alpha}$, and $\omega_{\alpha}$.

Due to the structure of the system (25), it is natural to consider reasonable assumptions. For formal simplicity, we describe diffusion relative to the barycentric frame, so that $\mathbf{v}=\mathbf{0}$, and observe that if the body force is due to gravity, then $\mathbf{b}_{\alpha}=\mathbf{b}=\mathbf{g}$, with $\mathbf{g}$ being the gravity acceleration, and thus $\mathbf{b}_{\alpha}-\mathbf{b}=\mathbf{0}$. Moreover, we consider the linear approximation and neglect

$$
(\mathbf{L}+\nabla \cdot \mathbf{v 1}) \mathbf{h}_{\alpha}, \quad \rho_{\alpha} \mathbf{u}_{\alpha} \otimes \mathbf{u}_{\alpha}
$$

Further, we take

$$
\mathbf{T}_{\boldsymbol{\alpha}}=-p_{\alpha} \mathbf{1}, \quad \mathrm{T}=-p \mathbf{1}, \quad p_{\alpha}=p \omega_{\alpha} .
$$

Accordingly, Equation (24) simplifies to

$$
\dot{\mathbf{h}}_{\alpha}=-p \nabla \omega_{\alpha}+\mathbf{m}_{\alpha}
$$

Observe that

$$
\overline{\nabla \cdot \mathbf{h}_{\alpha}}=\nabla \cdot \dot{\mathbf{h}}_{\alpha}-\mathbf{L h}_{\alpha} \simeq \nabla \cdot \dot{\mathbf{h}}_{\alpha}, \quad \overline{\rho \dot{\omega}_{\alpha}} \simeq \rho \ddot{\omega}_{\alpha}
$$

Hence, the time differentiation of (14) results in

$$
\rho \ddot{\omega}_{\alpha}=\nabla \cdot\left(p \nabla \omega_{\alpha}\right)+\nabla \cdot \mathbf{m}_{\alpha}+\dot{\tau}_{\alpha}
$$

It is reasonable to set

$$
\mathbf{m}_{\alpha}=\sum_{\beta} M_{\alpha \beta}\left(\mathbf{u}_{\beta}-\mathbf{u}_{\alpha}\right), \quad \tau_{\alpha}=\tau_{\alpha}\left(\omega_{1}, \omega_{2}, \ldots, \omega_{n}\right) .
$$

Hence, for reacting mixtures, the evolution of the mass fractions $\left\{\omega_{\alpha}\right\}$ is governed by a system of second-order differential equations for the $n$-tuple of mass fractions affected by the velocity differences $\mathbf{u}_{\beta}-\mathbf{u}_{\alpha}$.

It is worth considering the evolution of mixtures with two constituents. Let $\omega=\omega_{1}$ and hence $\omega_{2}=1-\omega$. Then, we have a single equation

$$
\rho \ddot{\omega}=\nabla \cdot(p \nabla \omega)+M \nabla \cdot\left(\mathbf{u}_{2}-\mathbf{u}_{1}\right)+f(\omega, \dot{\omega}) .
$$


Apart from the velocity difference $\mathbf{u}_{2}-\mathbf{u}_{1}$, the unknown mass fraction $\omega$ satisfies a nonlinear hyperbolic equation, with the ratio $p / \rho$ being the speed of propagation.

\section{A Continuum Derivation of Fick's Law}

In practical applications, the use of Equation (24) is quite difficult considering the occurrence of $L$ and the fact that they are a coupled system of equations. Again, we consider reasonable approximations. In addition, it is natural to know whether and how dynamic equations justify the use of Fick's law.

First, we assume that $\mathbf{b}_{\alpha}=\mathbf{b}$, as happens for gravity. Moreover, we select the barycentric reference and hence take $\mathbf{v}=\mathbf{0}$ and also $\mathbf{L}=\mathbf{0}$. Further, we neglect the nonlinear term $\rho_{\alpha} \mathbf{u}_{\alpha} \otimes$ $\mathbf{u}_{\alpha}$. The growth $\mathbf{m}_{\alpha}$ is assumed to be given by the velocity differences between constituents and it is proportional to the respective densities. Let

$$
\mathbf{m}_{\alpha}=\xi \rho_{\alpha} \sum_{\beta=1}^{n} \rho_{\beta}\left(\mathbf{v}_{\beta}-\mathbf{v}_{\alpha}\right),
$$

with $\xi$ being possibly a function of the temperature $\theta$. Since

$$
\sum_{\alpha=1}^{n} \mathbf{m}_{\alpha}=\xi \rho_{\alpha} \sum_{a, \beta=1}^{n} \rho_{\alpha} \rho_{\beta}\left(\mathbf{v}_{\beta}-\mathbf{v}_{\alpha}\right)=\mathbf{0}
$$

constraint (15) is identically satisfied. Moreover, we can write $\mathbf{m}_{\alpha}$ in the form

$$
\mathbf{m}_{\alpha}=\xi \rho_{\alpha} \sum_{\beta=1}^{n} \rho_{\beta}\left(\mathbf{u}_{\beta}-\mathbf{u}_{\alpha}\right) .
$$

We now restrict attention to binary mixtures. Hence, $\mathbf{h}_{1}+\mathbf{h}_{2}=\mathbf{0}$ and Equation (28) implies

$$
\mathbf{m}_{1}=\xi \rho_{1} \rho_{2}\left(\mathbf{u}_{2}-\mathbf{u}_{1}\right)=\xi\left(\rho_{1} \mathbf{h}_{2}-\rho_{2} \mathbf{h}_{1}\right)=-\xi \rho \mathbf{h}_{1}
$$

and

$$
\mathbf{m}_{2}=-\xi \rho \mathbf{h}_{2}
$$

As a consequence, Equation (24) becomes

$$
\dot{\mathbf{h}}_{\alpha}+\xi \rho \mathbf{h}_{\alpha}=\nabla \cdot \mathbf{T}_{\alpha}-\omega_{\alpha} \nabla \cdot \mathbf{T} .
$$

Further approximations are considered by restricting attention to stationary conditions, $\dot{\mathbf{h}}_{\alpha}=$ $\mathbf{0}$, and considering the constituents as inviscid fluids so that

$$
\mathbf{T}_{\alpha}=-p_{\alpha} 1, \quad \mathbf{T} \simeq-p 1, \quad p_{\alpha}=\omega_{\alpha} p .
$$

Hence, Equation (29) reduces to 


$$
\mathbf{h}_{\alpha}=\frac{p}{\xi \rho} \nabla \omega_{\alpha}
$$

which is just Fick's law for $\mathbf{h}_{\alpha}$, where $\kappa_{\alpha}=p / \xi \rho$.

\section{A Thermodynamic Justification of Assumption (17)}

It is interesting to show that assumption (17), which is the basis of the Cahn-Hilliard equation, is seemingly consistent with thermodynamics; it follows as a thermodynamic restriction if we ignore the dynamic property (24) of the diffusion fluxes $\left\{\mathbf{h}_{\alpha}\right\}$ within an approximated dynamic scheme.

The mixture is considered for a whole body and hence the balance of energy is taken in the form

$$
\rho \dot{\varepsilon}=\mathbf{T} \cdot \mathbf{D}-\nabla \cdot \mathbf{q}+\rho r
$$

where $\varepsilon$ is the internal energy density (per unit mass), $\boldsymbol{D}$ is the stretching, $\mathbf{q}$ is the heat flux vector, and $r$ is the external heat supply. The second law of inequality is assumed in the form

$$
\rho \dot{\eta}+\nabla \cdot \mathbf{j}-\frac{\rho r}{\theta} \geq 0
$$

where $\mathbf{j}$ is the entropy flux to be determined so that the inequality holds depending on the set of constitutive equations. For generality, let

$$
\mathbf{j}=\frac{\mathbf{q}}{\theta}+\mathbf{k}
$$

with $\mathbf{k}$ being the extra-entropy flux to be determined. Substituting $\rho r-\nabla \cdot \mathbf{q}$ from Equation (31), we have

$$
-\rho \dot{\varepsilon}+\rho \theta \dot{\eta}+\mathbf{T} \cdot \mathbf{D}-\frac{1}{\theta} \mathbf{q} \cdot \nabla \theta+\theta \nabla \cdot \mathbf{k} \geq 0
$$

Let $\psi=\varepsilon-\theta \eta$ be the Helmholtz free energy. The second law of inequality becomes

$$
-\rho(\dot{\psi}+\eta \dot{\theta})+\mathbf{T} \cdot \mathbf{D}-\frac{1}{\theta} \mathbf{q} \cdot \nabla \theta+\theta \nabla \cdot \mathbf{k} \geq 0
$$

Let

$$
\Xi=\left(\theta, \rho,\left\{\omega_{\alpha}\right\}, \nabla \theta, \nabla \rho,\left\{\nabla \omega_{\alpha}\right\}, \mathbf{D}, \dot{\theta}, \dot{\rho},\left\{\dot{\omega}_{\alpha}\right\}\right)
$$

be the set of independent variables, e.g., $\psi=\psi\left(\theta, \rho,\left\{\omega_{\alpha}\right\}, \nabla \theta, \nabla \rho,\left\{\nabla \omega_{\alpha}\right\}, \mathbf{D}, \dot{\rho},\left\{\dot{\omega}_{\alpha}\right\}\right)$. Moreover, let $\mathbf{T}=-p(\theta, \rho, \nabla \rho) \mathbf{1}+\boldsymbol{T}\left(\theta, \rho,\left\{\omega_{\alpha}\right\}, \nabla \theta, \nabla \rho,\left\{\nabla \omega_{\alpha}\right\}, \mathbf{D}, \dot{\theta}, \dot{\rho},\left\{\dot{\omega}_{\alpha}\right\}\right)$ with $\boldsymbol{T}=O(\mathbf{D}) \quad$ as $\quad \mathbf{D}$ approaches zero.

Substituting the time derivative $\dot{\psi}$, we obtain 


$$
\begin{gathered}
-\rho\left(\partial_{\theta} \psi+\eta\right) \dot{\theta}-\rho \partial_{\rho} \psi \dot{\rho}-\rho \sum_{\alpha=1}^{n}\left\{\partial_{\omega_{\alpha}} \psi \cdot \dot{\omega}_{\alpha}+\partial_{\nabla \omega_{\alpha}} \psi \cdot \dot{\overline{\nabla \omega_{\alpha}}}+\partial_{\dot{\omega}_{\alpha}} \psi \ddot{\omega}_{\alpha}\right\}-\rho \partial_{\nabla \theta} \psi \cdot \dot{\bar{\nabla} \theta}-\rho \partial_{\nabla \rho} \psi \cdot \dot{\bar{\nabla} \rho} \\
-\rho \partial_{\mathrm{D}} \psi \cdot(\dot{\boldsymbol{D}})-\rho \partial_{\theta} \psi \ddot{\theta}-\rho \partial_{\dot{\rho}} \psi \cdot \ddot{\rho}-p \nabla \cdot \mathbf{v}+\boldsymbol{\mathcal { T }} \cdot \mathbf{D}-\frac{1}{\theta} \mathbf{q} \cdot \nabla \theta+\theta \nabla \cdot \mathbf{k} \geq 0
\end{gathered}
$$

The arbitrariness and linearity of $\ddot{\omega}_{\alpha}, \ddot{\rho}, \ddot{\mathbf{D}}, \ddot{\theta}$ imply

$$
\psi=\psi\left(\theta, \rho,\left\{\omega_{\alpha}\right\}, \nabla \theta, \nabla \rho,\left\{\nabla \omega_{\alpha}\right\}\right)
$$

and hence

$$
\begin{gathered}
-\rho\left(\partial_{\theta} \psi+\eta\right) \dot{\theta}-\rho \partial_{\nabla \theta} \psi \cdot \dot{\overline{\nabla \theta}}-\rho \partial_{\rho} \psi \dot{\rho}-\rho \partial_{\nabla \rho} \psi \cdot \dot{\overline{\nabla \rho}} \\
-\rho \sum_{\alpha=1}^{n}\left\{\partial_{\omega_{\alpha}} \psi \cdot \dot{\omega}_{\alpha}+\partial_{\nabla \omega_{\alpha}} \psi \cdot \dot{\overline{\nabla \omega_{\alpha}}}\right\}-p \nabla \cdot \mathbf{v}+\boldsymbol{T} \cdot \mathbf{D}-\frac{1}{\theta} \mathbf{q} \cdot \nabla \theta+\theta \nabla \cdot \mathbf{k} \geq 0
\end{gathered}
$$

Observe

$$
\dot{\overline{\nabla \rho}}=\nabla \dot{\rho}-\mathrm{L}^{T} \nabla \rho
$$

and the analog for $\nabla \theta$ and $\nabla \omega_{\alpha}$. Then, divide it by $\theta$ and consider the identities

$$
\begin{aligned}
-\frac{\rho}{\theta} \partial_{\nabla \rho} \psi \cdot \dot{\bar{\nabla} \rho} & =-\frac{\rho}{\theta} \partial_{\nabla \rho} \psi \cdot \nabla \dot{\rho}+\frac{\rho}{\theta}\left(\nabla \rho \otimes \partial_{\nabla \rho} \psi\right) \cdot \mathrm{L} \\
& =-\nabla \cdot\left(\frac{\rho}{\theta} \partial_{\nabla \rho} \psi \rho\right)+\dot{\rho} \nabla \cdot\left(\frac{\rho}{\theta} \partial_{\nabla \rho} \psi\right)+\frac{\rho}{\theta}\left(\nabla \rho \otimes \partial_{\nabla \rho} \psi\right) \cdot \mathrm{L}
\end{aligned}
$$

and the same is for $\theta$. Moreover,

$$
\begin{aligned}
-\frac{\rho}{\theta} \partial_{\omega_{\alpha}} \psi \cdot \dot{\omega}_{\alpha} & -\frac{\rho}{\theta} \partial_{\nabla \omega_{\alpha}} \psi \cdot\left(\nabla \dot{\omega}_{\alpha}-\mathbf{L}^{T} \nabla \omega_{\alpha}\right) \\
& =-\frac{\rho}{\theta} \mu_{\alpha} \dot{\omega}_{\alpha}-\nabla \cdot\left(\frac{\rho}{\theta} \partial_{\omega_{\alpha}} \psi \dot{\omega}_{\alpha}\right)+\frac{\rho}{\theta}\left(\nabla \omega_{\alpha} \otimes \partial_{\nabla \omega_{\alpha}} \psi\right) \cdot \mathbf{L},
\end{aligned}
$$

where

$$
\mu_{\alpha}:=\partial_{\omega_{\alpha}} \psi-\frac{\theta}{\rho} \nabla \cdot\left(\frac{\rho}{\theta} \partial_{\nabla \omega_{\alpha}} \psi\right)
$$

Inequality (32) can then be written in the form

$$
\begin{gathered}
-\frac{\rho}{\theta}\left(\delta_{\theta} \psi+\eta\right) \dot{\theta}-\frac{\rho}{\theta} \sum_{\alpha=1}^{n} \mu_{\alpha} \dot{\omega}_{\alpha}+\frac{\rho}{\theta}\left(\nabla \theta \otimes \partial_{\nabla \theta} \psi+\nabla \rho \otimes \partial_{\nabla \rho} \psi+\sum_{\alpha=1}^{n} \nabla \omega_{\alpha} \otimes \partial_{\nabla \omega_{\alpha}} \psi\right) \cdot \mathrm{L} \\
-\frac{\rho}{\theta} \delta_{\rho} \psi \dot{\rho}-\frac{p}{\theta} \nabla \cdot \mathbf{v}+\frac{1}{\theta} \mathcal{T} \cdot \mathbf{D}-\frac{1}{\theta^{2}} \mathbf{q} \cdot \nabla \theta+\nabla \cdot\left(\mathbf{k}-\frac{\rho}{\theta} \partial_{\nabla \theta} \psi \dot{\theta}-\frac{\rho}{\theta} \partial_{\nabla \rho} \psi \dot{\rho}-\frac{\rho}{\theta} \sum_{\alpha=1}^{n} \partial_{\omega_{\alpha}} \psi \dot{\omega}_{\alpha}\right) \geq 0 .
\end{gathered}
$$

where 


$$
\delta_{\theta} \psi:=\partial_{\theta} \psi-\frac{\theta}{\rho} \nabla \cdot\left(\frac{\rho}{\theta} \partial_{\nabla \theta} \psi\right), \delta_{\rho} \psi:=\partial_{\rho} \psi-\frac{\theta}{\rho} \nabla \cdot\left(\frac{\rho}{\theta} \partial_{\nabla \rho} \psi\right)
$$

Since $\mathbf{L}=\mathbf{D}+\mathbf{W}, \mathbf{W} \in \mathrm{Skw}$ is the (skew) spin, then the arbitrariness of $W$ implies

$$
\nabla \theta \otimes \partial_{\nabla \theta} \psi+\nabla \rho \otimes \partial_{\nabla \rho} \psi+\sum_{\alpha=1}^{n} \nabla \omega_{\alpha} \otimes \partial_{\nabla \omega_{\alpha}} \psi \in \text { Sym. }
$$

Hence,

$$
\widehat{\mathbf{T}}=\boldsymbol{T}+\nabla \theta \otimes \partial_{\nabla \theta} \psi+\nabla \rho \otimes \partial_{\nabla \rho} \psi+\sum_{\alpha=1}^{n} \nabla \omega_{\alpha} \otimes \partial_{\nabla \omega_{\alpha}} \psi \in \text { Sym. }
$$

Moreover, since $\dot{\rho}=-\rho \nabla \cdot \mathbf{v}$, then let

$$
p=\rho^{2} \delta_{\rho} \psi
$$

Then, in light of Equation (14) we replace $\rho \dot{\omega}_{\alpha}$ with $-\nabla \cdot \mathbf{h}_{\alpha}+\tau_{\alpha}$ and observe

$$
-\frac{\rho}{\theta} \sum_{\alpha=1}^{n} \mu_{\alpha} \dot{\omega}_{\alpha}=\frac{1}{\theta} \sum_{\alpha=1}^{n} \mu_{\alpha} \nabla \cdot \mathbf{h}_{\alpha}-\frac{1}{\theta} \sum_{\alpha=1}^{n} \mu_{\alpha} \tau_{\alpha}=\nabla \cdot \sum_{\alpha=1}^{n} \frac{\mu_{\alpha}}{\theta} \mathbf{h}_{\alpha}-\sum_{\alpha=1}^{n} \mathbf{h}_{\alpha} \cdot \nabla \frac{\mu_{\alpha}}{\theta}-\frac{1}{\theta} \sum_{\alpha=1}^{n} \mu_{\alpha} \tau_{\alpha} .
$$

Hence, we can write

$$
\begin{aligned}
& -\frac{\rho}{\theta}\left(\delta_{\theta} \psi+\eta\right) \dot{\theta}-\sum_{\alpha=1}^{n} \mathbf{h}_{\alpha} \cdot \nabla \frac{\mu_{\alpha}}{\theta}-\frac{1}{\theta} \sum_{\alpha=1}^{n} \mu_{\alpha} \tau_{\alpha}+\frac{1}{\theta} \mathbf{T} \cdot \mathbf{D}-\frac{1}{\theta^{2}} \mathbf{q} \cdot \nabla \theta \\
& +\nabla \cdot\left(\mathbf{k}-\frac{\rho}{\theta} \partial_{\nabla \theta} \psi \dot{\theta}-\frac{\rho}{\theta} \partial_{\nabla \rho} \psi \dot{\rho}-\frac{\rho}{\theta} \sum_{\alpha=1}^{n} \partial_{\omega_{\alpha}} \psi \dot{\omega}_{\alpha}+\sum_{\alpha=1}^{n} \frac{\mu_{\alpha}}{\theta} \mathbf{h}_{\alpha}\right) \geq 0
\end{aligned}
$$

Inequality (33) hold if

$$
\eta=-\delta_{\theta} \psi, \quad \mathbf{k}=\frac{\rho}{\theta} \partial_{\nabla \theta} \psi \dot{\theta}+\frac{\rho}{\theta} \partial_{\nabla \rho} \psi \dot{\rho}+\frac{\rho}{\theta} \sum_{\alpha=1}^{n} \partial_{\omega_{\alpha}} \psi \dot{\omega}_{\alpha}-\sum_{\alpha=1}^{n} \frac{\mu_{\alpha}}{\theta} \mathbf{h}_{\alpha}
$$

and

$$
-\sum_{\alpha=1}^{n} \mathbf{h}_{\alpha} \cdot \nabla \frac{\mu_{\alpha}}{\theta}-\frac{1}{\theta} \sum_{\alpha=1}^{n} \mu_{\alpha} \tau_{\alpha}+\frac{1}{\theta} \widehat{\mathbf{T}} \cdot \mathbf{D}-\frac{1}{\theta^{2}} \mathbf{q} \cdot \nabla \theta \geq 0
$$

Since $\sum_{\alpha=1}^{n} \mathbf{h}_{\alpha}=\mathbf{0}$ and $\sum_{\alpha=1}^{n} \tau_{\alpha}=0$, then

$$
\sum_{\alpha=1}^{n} \mathbf{h}_{\alpha} \cdot \nabla \frac{\mu_{\alpha}}{\theta}=\sum_{\alpha=1}^{n-1} \mathbf{h}_{\alpha} \cdot \nabla \frac{\mu_{\alpha}-\mu_{n}}{\theta}, \sum_{\alpha=1}^{n} \mu_{\alpha} \tau_{\alpha}=\sum_{\alpha=1}^{n-1}\left(\mu_{\alpha}-\mu_{n}\right) \tau_{\alpha}
$$


Setting aside cross-coupling effects, we conclude that inequality (34) holds if the single terms are non-negative. The first term of Equation (34) is non-negative if

$$
\mathrm{h}_{\alpha}=-\hat{\kappa}_{\alpha} \nabla \frac{\mu_{\alpha}-\mu_{n}}{\theta}, \quad \hat{\kappa}_{\alpha} \geq 0, \quad \alpha=1, \ldots, n-1 .
$$

Equation (35) is analogous to Equation (17) of classical and quantum models under isothermal conditions.

The present derivation, however, shows the weak point of the assumption. The theory of mixtures shows that the free energy of the mixture as a whole is given by

$$
\psi=\sum_{\alpha=1}^{n} \omega_{\alpha}\left(\psi_{\alpha}+\frac{1}{2} \mathbf{u}_{\alpha}^{2}\right)
$$

The derivation instead is consistent with the assumption that

$$
\psi=\sum_{\alpha=1}^{n} \psi_{\alpha}, \quad \psi_{\alpha}=\psi_{\alpha}\left(\theta, \rho_{\alpha}, \nabla \rho_{\alpha}\right), \quad \rho_{\alpha}=\rho \omega_{\alpha}
$$

Neglecting $\mathbf{u}_{\alpha}^{2} / 2$ eliminates a dynamic term that is considered essential in the diffusion process.

\section{Conclusions and Review of Classical Diffusion Equations}

According to the Schrödinger equation, quantum models of diffusion are naturally related to the selection of the potential. Further, a close analogy is observed between the classical models where the diffusion flux in a mixture is related to the chemical potential. The resulting differential equation for the mass fraction $\omega$ is qualitatively dependent on the chemical potential function of $\omega$ or $\omega, \nabla \omega$. The classical diffusion equation is formally equal to the heat equation, whereas other models lead to more involved differential equations that are still of parabolic character. The following table shows how the main approaches of the literature are used to derive the pertinent diffusion equations, with the same notation as discussed in this paper.

\begin{tabular}{lcc}
\hline & Assumption & Diffusion equation \\
\hline Fick & $\mathbf{h}_{\alpha}=-D \nabla \omega_{\alpha}$ & $\partial_{t} \omega_{\alpha}=D \Delta \omega_{\alpha}$ \\
Cahn-Hilliard & $\partial_{t} \omega=-\nabla \cdot \mathbf{h}=\kappa \nabla \delta_{\omega} \psi$ & $\partial_{t} \omega=\kappa \Delta\left(\partial_{\omega} \psi-\gamma \Delta \omega\right)$ \\
Cahn-Allen & $\partial_{t} \omega=-k \delta_{\omega} \psi$ & $\partial_{t} \omega=k \nabla \cdot\left(\gamma \nabla \omega-\frac{1}{2} \partial_{\omega} \gamma|\nabla \omega|^{2}-\partial_{\omega} \psi\right)$ \\
Nernst-Planck & $\mathbf{h}=-D\left(\nabla \omega+\left(\frac{q}{k_{B} \theta}\right) \omega \nabla \phi\right)$ & $\partial_{t} \omega=D \nabla \cdot\left(\nabla \omega+\left(q / k_{B} \theta\right) \omega \nabla \phi\right)$ \\
Onsager & $\mathbf{h}_{\alpha}=-\sum_{\beta} L_{\alpha \beta} \nabla \mu_{\beta}$ & $\partial_{t} \omega_{\alpha}=\nabla \cdot\left(\sum_{\beta} D_{\alpha \beta} \omega_{\beta}\right)$ \\
Rescaled potential & $\mathbf{h}=-k \nabla(\mu / \theta)-l \nabla(1 / \theta)$ & $\partial_{t} \omega=-\nabla \cdot \mathbf{h}$ \\
\hline
\end{tabular}

Though the theory of mixture shows that the correct dynamics is governed by Equation (14), the mass density $\rho$ is quite often ignored or considered as a constant. In essence, the Cahn-Hilliard 
and Cahn-Allen (or Ginzburg-Landau) equations [2] derive the evolution equations considering that the free energy $\psi$ depends on both $\omega$ and $\nabla \omega$. Moreover, it is assumed that $\partial_{\omega} \psi$ is given by the variational derivative $\delta_{\omega} \psi=\partial_{\omega} \psi-\nabla \cdot \partial_{\nabla \omega} \psi$. The Nernst-Planck model [25-27] describes the diffusion of ions and generalizes Fick's model by adding the contribution of the electric potential $\phi$, where $q$ denotes the ionic charge and $k_{B}$ is the Boltzmann constant. The definition (13) of the diffusion flux $\mathbf{h}_{\boldsymbol{\alpha}}$ is used to determine the diffusion velocity $\mathbf{u}_{\boldsymbol{\alpha}}$ once the expression of $\mathbf{h}_{\boldsymbol{\alpha}}$ is assumed. This is, e.g., the case of earlier studies [28] for the diffusion velocity in terms of the Nernst-Planck flux. In an earlier study [29], Onsager's relation of the diffusion flux is considered as a linear combination of forces next identified with the gradient of chemical potentials. The above view is generalized in earlier studies [30] by considering the flux as a linear combination of concentration gradients. The definition of the flux in terms of the rescaled chemical potential $\mu / \theta$ mainly occurs in classical models [31]. This view is found to be consistent with the result of Equation (35).

In this paper, it is emphasized that a correct model of diffusion should be based on the dynamics (continuity equations and equations of motion) of a mixture. Further, it is shown that how appropriate approximations (linearizations) lead to known models of diffusion. Conceptually, the evolution equation (24), characterizing the diffusion fluxes, is tacitly ignored in known models.

It is worth mentioning that the diffusion models analyzed in this paper are suitable for a system whose space size is in nanoscale. In such cases, constitutive equations that involve the density (or mass fraction) gradient in addition to the density should be considered. This principle is extensively discussed in earlier studies [32] in connection with confinement and tunneling in semiconductor devices. Hence, the balance equations remain unchanged, and the constitutive dependence should exhibit dependence on the density gradients.

\section{Author Contributions}

The author did all the research work of this study.

\section{Competing Interests}

The author has declared that no competing interests exist.

\section{References}

1. De Falco C, Jerome JW, Sacco R. Quantum-corrected drift-diffusion models: Solution fixed point map and finite element approximation. J Comput Phys. 2009; 228: 1770-1789.

2. Brokate $M$, Sprekels J. Hysteresis and phase transitions. New York: Springer Science \& Business Media; 1996.

3. Echebarria B, Folch R, Karma A, Plapp M. Quantitative phase-field model of alloy solidification. Phys Rev E. 2004; 70: 061604.

4. Klika V, Maršík F. Coupling effect between mechanical loading and chemical reactions. J Phys Chem B. 2009; 113: 14689-14697.

5. Jabbour ME, Bhattacharya K. A continuum theory of multispecies thin solid film growth by chemical vapor deposition. J Elast. 2003; 73: 13-74.

6. Bothe D. On the Maxwell-Stefan approach to multicomponent diffusion. In Parabolic problems. Basel: Springer; 2011. pp.81-93.

7. Madelung E. Quantum theory in hydrodynamical form. Zeit $f$ Phys. 1927; 40: 322-325. 
8. Bohm D. A suggested interpretation of the quantum theory in terms of "hidden" variables. I. Phys Rev. 1952; 85: 166-179.

9. Beasley MR. Notes on the Ginzburg-Landau Theory. ICMR Summer School on Novel Superconductors. Santa Barbara, CA: University of California; 2009.

10. Schuller IK, Gray KE. Time-dependent Ginzburg-Landau: From single particle to collective behavior. J Supercond Nov Magn. 2006; 19: 401-407.

11. Tsekov R. Thermo-quantum diffusion. Int J Theor Phys. 2009; 48: 630-636.

12. Nassar AB. Fluid formulation of a generalised Schrodinger-Langevin equation. J Phys A Math Gen. 1985; 18: L509-L511.

13. Ancona MG, lafrate GJ. Quantum correction to the equation of state of an electron gas in a semiconductor. Phys Rev B. 1989; 39: 9536-9540.

14. Tsekov R. Quantum diffusion. Phys Scr. 2011; 83: 035004.

15. Degond $P$, Méhats $F$, Ringhofer $C$. Quantum energy-transport and drift-diffusion models. J Stat Phys. 2005; 118: 625-667.

16. Mueller I. Thermodynamics of mixtures of fluids. J de Mec. 1975; 14: 267-303.

17. Morro A. Diffusion in mixtures of reacting thermoelastic solids. J Elast. 2016; 123: 59-84.

18. Fick A. Ueber diffusion. Ann Phys. 1855; 170: 59-86.

19. Cahn JW. On spinodal decomposition. Acta Metall. 1961; 9: 795-801.

20. Cahn JW, Hilliard JE. Spinodal decomposition: A reprise. Acta Metall. 1971; 19: 151-161.

21. Allen SM, Cahn JW. A microscopic theory for antiphase boundary motion and its application to antiphase domain coarsening. Acta Metall. 1979; 27: 1085-1095.

22. Chan SK. Steady-state kinetics of diffusionless first order phase transformations. J Chem Phys. 1977; 67: 5755-5762.

23. Müller I. Thermodynamics of mixtures and phase field theory. Int J Solids Struct. 2001; 38: 1105-1113.

24. Morro A. Balance and constitutive equations for diffusion in mixtures of fluids. Meccanica. 2014; 49: 2109-2123.

25. Nernst W. Zur kinetik der in lösung befindlichen körper. Z phys Chem. 1888; 2: 613-637.

26. Nernst W. Die elektromotorische wirksamkeit der jonen. Z Phys Chem. 1889; 4: 129-181.

27. Planck M. Ueber die erregung von electricität und wärme in electrolyten. Ann Phys Chem. 1890; 275: 161-186.

28. Danielewski M, Wierzba B. Thermodynamically consistent bi-velocity mass transport phenomenology. Acta Mater. 2010; 58: 6717-6727.

29. Ågren J. Diffusion in phases with several components and sublattices. J Phys Chem Solids. 1982; 43: 421-430.

30. Andersson JO, Ågren J. Models for numerical treatment of multicomponent diffusion in simple phases. J Appl Phys. 1992; 72: 1350-1355.

31. Alt HW, Pawlow I. A mathematical model of dynamics of non-isothermal phase separation. Physica D. 1992; 59: 389-416.

32. Ancona MG. Density-gradient theory: A macroscopic approach to quantum confinement and tunneling in semiconductor devices. J Comput Electron. 2011; 10: 65-97. 


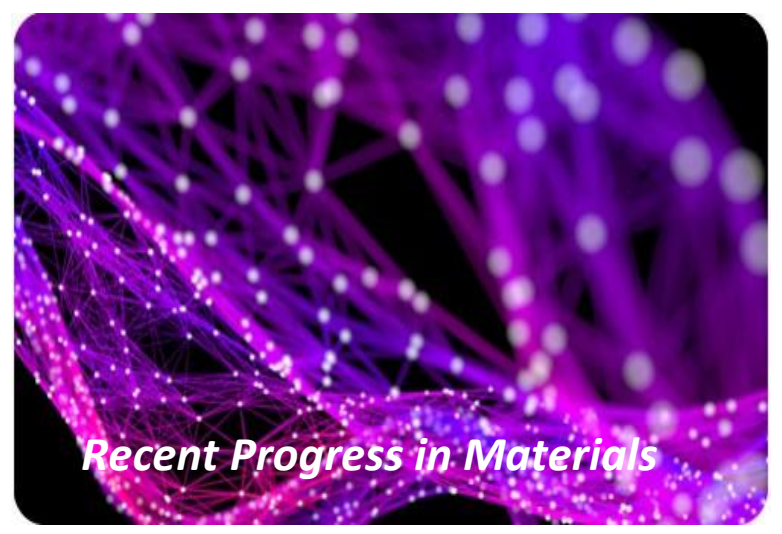

Enjoy Recent Progress in Materials by:

1. Submitting a manuscript

2. Joining in volunteer reviewer bank

3. Joining Editorial Board

4. Guest editing a special issue

For more details, please visit:

http://www.lidsen.com/journals/rpm 